\title{
VALIDAÇÃO E ESTIMATIVA DA INCERTEZA DE MÉTODO PARA ANÁLISE DE LICOPENO E B-CAROTENO EM POLPA DE TOMATE POR CROMATOGRAFIA LÍQUIDA DE ALTA EFICIÊNCIA
}

\author{
Adriana Barreto Alves, Marta Gomes da Silva*, Paulo Roberto Nogueira Carvalho e Lizziane Cynara Vissotto \\ Centro de Ciência e Qualidade de Alimentos, Instituto de Tecnologia de Alimentos, CP 139, 13070-178 Campinas - SP, Brasil \\ Neura Bragagnolo
}

Departamento de Ciência de Alimentos, Faculdade de Engenharia de Alimentos, Universidade Estadual de Campinas, CP 6121, 13083-862 Campinas - SP, Brasil

Recebido em 6/11/09; aceito em 28/4/10; publicado na web em 9/8/10

\begin{abstract}
VALIDATION AND UNCERTAINTY ESTIMATION OF A METHOD BY HIGH PERFORMANCE LIQUID CHROMATOGRAPHY FOR THE ANALYSIS OF LYCOPENE AND $\beta$-CAROTENE IN TOMATO PULP. A method to quantify lycopene and $\beta$-carotene in freeze dried tomato pulp by high performance liquid chromatography (HLPC) was validated according to the criteria of selectivity, sensitivity, precision and accuracy, and uncertainty estimation of measurement was determined with data obtained in the validation. The validated method presented is selective in terms of analysis, and it had a good precision and accuracy.

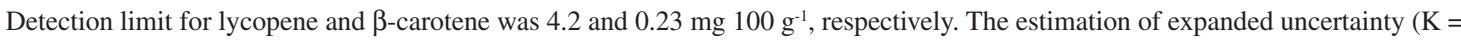
2) for lycopene was $104 \pm 21 \mathrm{mg} 100 \mathrm{~g} \mathrm{~g}^{-1}$ and for $\beta$-carotene was $6.4 \pm 1.5 \mathrm{mg} 100 \mathrm{~g}^{-1}$.
\end{abstract}

Keywords: tomato pulp; validation; uncertainty estimation.

\section{INTRODUÇÃO}

O tomate (Lycoperson esculentum) é um fruto originário das Américas, sazonal, disponível por apenas 3 ou 4 meses ao ano. ${ }^{1} \mathrm{O}$ Brasil é o $9^{\circ}$ produtor mundial de tomates, sendo os estados de São Paulo, Minas Gerais e Goiás responsáveis por aproximadamente $65 \%$ da produção nacional. Em 1850, o tomate começou a ser industrializado e no início do século XX a produção de tomate tornou-se um ramo industrial extremamente rentável e bastante popular no mercado na forma de polpa concentrada ou extrato de tomate. ${ }^{2} \mathrm{O}$ tomate caracteriza-se por possuir alto teor de licopeno, responsável pela cor vermelha em seus produtos, $\beta$-caroteno, que apresenta atividade pró-vitamínica A, e outros carotenoides em menor concentração. ${ }^{3}$

Embora o Food and Drug Administration (FDA - USA) considere limitada a evidência de associação do consumo de tomates e a redução do risco de câncer de próstata, ovário, gástrico e pâncreas, ${ }^{4}$ os carotenoides estão relacionados com ação antioxidante e controle ou prevenção de doenças crônicas. ${ }^{5}$

Segundo Carvalho, ${ }^{2}$ o Brasil apresenta expansão da cultura de tomates e a indústria de processamento tem acompanhado o crescimento, o que torna necessário um controle mais eficaz das etapas do processamento, bem como dos produtos obtidos. Desta forma, se fazem necessários métodos validados e com a estimativa de incerteza calculada, para acompanhar o rendimento e degradação de carotenos durante as etapas de processamento e estocagem de produtos de tomate.

No processo de validação de um método, a estimativa da incerteza é o principal foco de interesse devido a sua importância em mostrar a qualidade dos dados. O Guia EURACHEM/CITAC ${ }^{6}$ define incerteza como "um parâmetro associado ao resultado de uma medição, que caracteriza a dispersão de valores que poderiam ser razoavelmente atribuídos ao mensurando". Para a maioria dos casos, a incerteza expandida $(U)$ deve ser utilizada. $U$ é obtida pela multiplicação da incerteza padrão combinada $(\mu)$ por um fator de abrangência $(K)$. A escolha do fator $K$ é baseada no nível de confiança desejado. ${ }^{6}$ Dentre

*e-mail: martags@ital.sp.gov.br as técnicas analíticas para determinar carotenoides, é recomendada a cromatografia líquida de alta eficiência com detector de arranjo de diodos (CLAE-DAD), por permitir monitoração simultânea de vários compostos, além de fornecer o espectro de absorção característico do cromóforo e a pureza do pico. ${ }^{6}$

Dentro deste panorama, o objetivo deste trabalho foi validar um método por CLAE-DAD para determinar licopeno e $\beta$-caroteno em polpa de tomate liofilizada e determinar a estimativa de incerteza expandida de medição atribuída ao método proposto.

\section{PARTE EXPERIMENTAL}

\section{Amostras}

As amostras utilizadas foram coletadas durante o processamento de polpa concentrada de tomate na empresa Predilecta Alimentos Ltda, localizada no município de Matão - SP. Para a validação do método, foi utilizada uma amostra de polpa concentrada de tomate obtida na etapa final do processamento (produto final) e outra do resíduo do processamento (pele + semente). As amostras foram liofilizadas e armazenadas a $-20^{\circ} \mathrm{C}$ até o momento da análise.

\section{Reagentes}

O padrão de licopeno foi obtido no laboratório a partir do extrato de uma amostra. O licopeno foi isolado do extrato de carotenoides por cromatografia em coluna aberta, tendo como adsorvente óxido de magnésio (Art Lab) e hyflosupercel (Synth) (1:2, p/p). A primeira fração de carotenoides foi eluída com éter de petróleo:éter etílico (7:3, v/v), seguida pela eluição do licopeno com acetona. A fração contendo licopeno foi seca sob nitrogênio e suspendida em éter de petróleo. A concentração foi determinada por espectrofotometria a $472 \mathrm{~nm}$ utilizando o coeficiente de absorção $\left(E_{1 \mathrm{~cm}}^{1 \%}\right)$ de 3450 (unidades de absorbância). A pureza do licopeno foi avaliada na mesma condição cromatográfica descrita para o processo analítico, usando-se o detector de arranjo de diodos (DAD). A pureza encontrada foi de $99 \%$. 
O padrão de $\beta$-caroteno utilizado foi o trans- $\beta$-caroteno $95 \%$ (Sigma, USA). Cerca de $25 \mathrm{mg}$ de trans- $\beta$-caroteno foram dissolvidos em $25 \mathrm{~mL}$ de éter de petróleo e a concentração foi corrigida por espectrofotometria a $453 \mathrm{~nm}$ com uso do coeficiente de absorção ${ }^{7}$ $\left(E^{1 \%}\right)$ de 2592 (unidades de absorbância).

Metanol, acetonitrila e acetato de etila utilizados no processo cromatográfico foram de grau cromatográfico (Tedia, USA) e demais reagentes utilizados foram de grau analítico.

\section{Equipamentos}

Foi utilizado um cromatógrafo líquido marca Shimadzu CLASS-10 com bomba binária, modelo LC10, equipado com detector de arranjo de diodos (DAD-UV-VIS), modelo SPD-M10A e alça amostradora de $250 \mu \mathrm{L}$. Na separação dos compostos foi utilizada coluna de fase reversa LiCrospher $100 \mathrm{RP} 18,5 \mu \mathrm{m}, 125 \mathrm{~mm}$ x 4 mm d.i. (Merck, Alemanha), com pré-coluna LiCrospher 100 RP 18, $5 \mu \mathrm{m}, 4$ x mm d.i. (Merck, Alemanha).

\section{Método analítico}

O método validado foi baseado no descrito por Carvalho et al.. ${ }^{8}$ Para a extração dos carotenoides, foram tomados cerca de $0,5 \mathrm{~g}$ de polpa de tomate liofilizada, adicionados cerca de $3 \mathrm{~g}$ de hyflosupercel, homogeneizado e hidratado com $20 \mathrm{~mL}$ de água. Os carotenoides foram extraídos, de forma sequencial, com uma porção de $50 \mathrm{~mL}$ e três porções de $30 \mathrm{~mL}$ de acetona, em desintegrador por $1 \mathrm{~min}$, até a descoloração da amostra. Alíquotas de $20 \mathrm{~mL}$ foram transferidas para um funil de separação contendo $50 \mathrm{~mL}$ de éter de petróleo, e a acetona foi eliminada pela partição com água. Após a eliminação da acetona, os carotenoides dissolvidos no éter de petróleo foram transferidos para balão volumétrico de $50 \mathrm{~mL}$ e o volume ajustado. Para a análise de $\beta$-caroteno, uma alíquota de $1 \mathrm{~mL}$ do extrato foi seca sob fluxo de nitrogênio e diluída em $1 \mathrm{~mL}$ de fase móvel. Para licopeno foram secos, pelo mesmo processo, $0,5 \mathrm{~mL}$ de amostra e diluída em $5 \mathrm{~mL}$ de fase móvel. As amostras diluídas na fase móvel foram colocadas em vórtex por 1 min e filtradas em membrana de celulose regenerada de $0,45 \mu \mathrm{m}$ (Millipore).

Os carotenoides foram separados em sistema de eluição isocrático, sendo a fase móvel constituída de acetonitrila:metanol:acetato de etila (73:20:07, v/v/v) com vazão de $1,5 \mathrm{~mL} \mathrm{~min}^{-1}$ e detecção a $450 \mathrm{~nm}$.

\section{Validação}

A validação do método foi realizada segundo os parâmetros do INMETRO,${ }^{9}$ CODEX,${ }^{10}$ e Quattrocchi et al.. ${ }^{11}$ Foram avaliados, na matriz polpa de tomate liofilizada, seletividade, sensibilidade, exatidão e precisão do processo analítico.

\section{Seletividade}

A seletividade foi avaliada pela pureza do pico de licopeno e $\beta$ caroteno em polpa de tomate (produto final), e pelo efeito da matriz na técnica proposta para a quantificação desses pigmentos. $\mathrm{O}$ efeito da matriz para o analito licopeno foi avaliado, na amostra de resíduo do processamento (pele + semente), sem adição do analito e com adição de 2,02 mg $100 \mathrm{~g}^{-1}$.Para o analito $\beta$-caroteno, foi utilizada a amostra polpa de tomate (produto final), sem a adição do analito e com adição de $0,55 \mathrm{mg} 100 \mathrm{~g}^{-1}$. O efeito da matriz foi avaliado, estatisticamente, com o teste $t$-Student, Equações 1 e 2 .

$$
\begin{gathered}
t=|\overline{X 1}-\overline{X 2}| / \sqrt{s^{2}(1 / n 1+1 / n 2)} \\
s^{2}=\left[(n 1-1) s^{2} 1+(n 2-1) s^{2} 2\right] /(n 1+n 2-1)
\end{gathered}
$$

onde: $\overline{X 1}$ e $\overline{X 2}$, médias das respostas dos analitos nas amostras com e sem adição dos mesmos; s1 e s2, estimativas de desvios-padrão da resposta desses analitos; $\mathrm{n} 1$ e $\mathrm{n} 2$, tamanhos das amostras 1 e 2 .

\section{Sensibilidade}

A sensibilidade foi avaliada com a construção de uma curva analítica composta pelos analitos licopeno e $\beta$-caroteno com seis concentrações considerando a origem. Para o licopeno, foram utili-

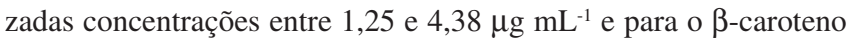
entre 0,37 e $1,65 \mu \mathrm{g} \mathrm{mL}{ }^{-1}$, diluídos na fase móvel. As estimativas de limite de detecção (LD) e limite de quantificação (LQ) foram obtidas com curvas analíticas suplementares, com três concentrações inferiores à menor concentração dos analitos, injetadas em triplicata. A linearidade da curva analítica e os LD e LQ foram obtidos com a aplicação das Equações 3, 4 e 5, respectivamente.

$$
t_{r}=|r| \sqrt{(n-2)} / \sqrt{\left(1-r^{2}\right)}
$$

$$
\begin{gathered}
\text { Limite de det ecção }=\left[\left(Y_{b l}+3 S_{b l}\right) / b\right](1 / \sqrt{n}) \\
\text { Limite de quantificação } \left.=\left[\left(Y_{b l}+10 S_{b l}\right) / b\right)\right](1 / \sqrt{n})
\end{gathered}
$$

onde: $t_{r}$, valor calculado para comparar com $t$-Student tabelado; $\mathrm{r}$, correlação linear; $\mathrm{n}$, número de medidas; $\mathrm{Y}_{\mathrm{b}}$, estimativa da resposta do branco (coeficiente linear obtido na equação da curva construída com a concentração do analito e a área do pico cromatográfico); $\mathrm{S}_{\mathrm{bl}}$, estimativa de desvio-padrão do branco (coeficiente linear obtido na equação da curva construída com a concentração do analito e a estimativa de desvio-padrão); b, coeficiente angular da curva analítica.

\section{Precisão}

A precisão do método foi avaliada com cinco repetições analíticas, simultâneas e independentes de uma amostra, realizadas com o mesmo método, técnico e equipamento com pequeno intervalo de tempo. A precisão do sistema foi obtida com cinco injeções consecutivas de um ponto da curva analítica. $\mathrm{O}$ coeficiente de variação $(\mathrm{CV})$ encontrado entre as repetições foi avaliado segundo Horwitz ${ }^{12}$ (Equação 6). Para essa parte do estudo foi utilizada a polpa de tomate concentrada (produto final).

$$
C V(\%)=2^{\left(1-0,5 \log _{10} C\right)}
$$

onde: C, concentração estudada expressa como potência de 10 .

\section{Exatidão}

Para avaliar a exatidão do método para o analito $\beta$-caroteno foi utilizada a amostra de polpa de tomate (produto final) por apresentar valor médio de $6,4 \mathrm{mg} 100 \mathrm{~g}^{-1}$, considerado baixo em relação ao teor médio de licopeno de $104 \mathrm{mg} 100 \mathrm{~g}^{-1}$. Desse modo, a concentração natural de $\beta$-caroteno não interferiu nos testes de recuperação. Para o teste de exatidão do método para o analito licopeno, foi selecionado o produto identificado como resíduo do processamento (pele + semente) por apresentar um teor médio de licopeno de $13,6 \mathrm{mg} 100 \mathrm{~g}^{-1}$, e pela dificuldade em se obter uma solução com concentração alta de licopeno. O teste de recuperação foi realizado em triplicata, com adição dos padrões em dois níveis

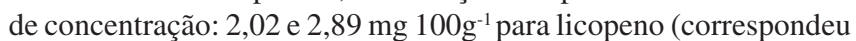
a 15 e $21 \%$ do teor encontrado na amostra teste) e 0,55 e $0,91 \mathrm{mg}$ $100 \mathrm{~g}^{-1}$ para $\beta$-caroteno (correspondeu a 9 e $14 \%$ do teor encontrado na amostra). Para avaliar estatisticamente o teste de exatidão foi utilizada a Equação 7.

$$
t_{c a l}=(|X-\bar{X}| / s) \sqrt{n}
$$


onde: $t_{\text {cal }}$, valor calculado para comparar com o $t$-Student tabelado; $X$, corresponde a $100 \% ; \bar{X}$, recuperação média; s, estimativa de desvio padrão das recuperações; $n$, número de medidas.

\section{Estimativa da incerteza}

Segundo o Vocabulário Internacional de Termos Fundamentais e Gerais de Metrologia - VIM, ${ }^{13}$ “incerteza de medição é o parâmetro, associado ao resultado de uma medição, que caracteriza a dispersão dos valores que podem ser fundamentadamente atribuídos a um mensurando". Na determinação de carotenoides por CLAE-DAD o resultado, concentração de carotenoide em mg $100 \mathrm{~g}^{-1}$, foi fornecido pela Equação 8. Logo, a incerteza combinada é a raiz quadrada da soma das incertezas ao quadrado da diluição da amostra, área da amostra, concentração do padrão, área do padrão e massa da amostra, Equação 9.

$$
\begin{gathered}
C_{c a r}=\left(\left(D_{i m} x A_{a m} x C_{P}\right) /\left(A_{P} x m_{a m}\right)\right) x 100 \\
\mu_{c} C_{c a r}=C_{c a r} \sqrt{\left(\frac{\mu D i l_{a m}}{D i l_{a m}}\right)^{2}+\left(\frac{\mu A_{a m}}{A_{a m}}\right)^{2}+\left(\frac{\mu C_{P}}{C_{p}}\right)^{2}+\left(\frac{\mu A_{P}}{A_{P}}\right)^{2}+\left(\frac{\mu m_{a m}}{m_{a m}}\right)^{2}}
\end{gathered}
$$

onde: $\mathrm{C}_{\text {car }}$, concentração do carotenoide na amostra; Dil, diluição da amostra; $A_{a m}$, área da amostra; $C_{p}$, concentração do padrão; $A_{p}$, área do padrão; $m$ é a massa da amostra.

\section{RESULTADOS E DISCUSSÃO}

\section{Seletividade}

A Figura 1 apresenta o cromatograma planar característico de uma amostra de polpa de tomate utilizada nesse estudo. A avaliação da pureza dos picos correspondentes ao licopeno e $\beta$-caroteno indicou uma pureza superior a $99 \%$. A pureza encontrada sugeriu uma seletividade adequada para a técnica utilizada para quantificar os analitos na matriz.

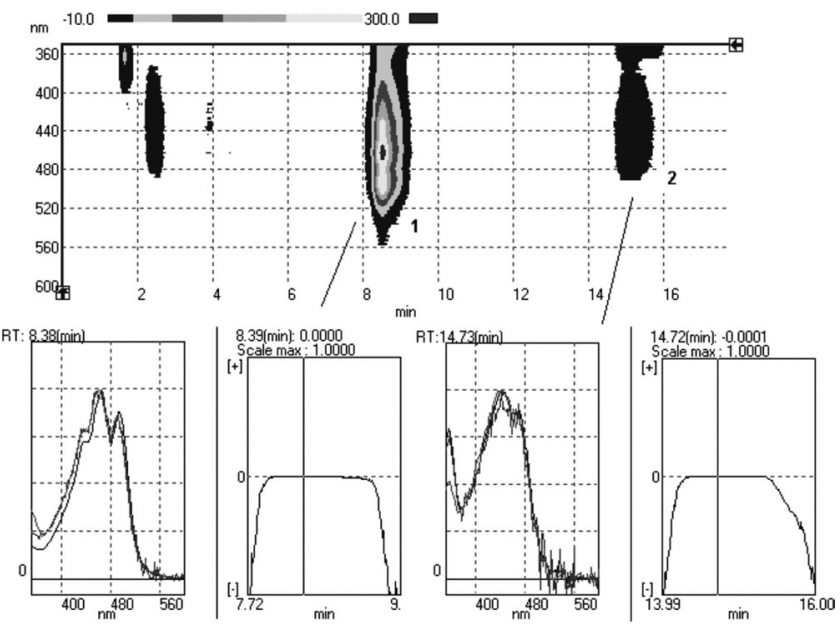

Figura 1. Cromatograma planar entre 350 e $600 \mathrm{~nm}$ de uma amostra de polpa de tomate, com a pureza de pico encontrada para licopeno (pico 1)e $\beta$-caroteno (pico 2). Condições de análise descritas no texto

Os dados utilizados para calcular o efeito da matriz estão apresentados na Tabela 1. Os valores $t$ calculados para o teste de seletividade foram comparados com o valor tabelado $(t$-Student $) \operatorname{com}(n 1+n 2-2)$ graus de liberdade para uma probabilidade de $95 \%\left(t\right.$ tabelado $_{(95 ; 4)}$ igual a 2,132). ${ }^{14}$ Os valores $t$ calculados para licopeno e $\beta$-caroteno foram 0,103 e 1,319, respectivamente. Os resultados indicam que a matriz não interfere na análise e o método apresenta seletividade adequada. Embora a quantificação para os dois analitos tenha ocorrido a $450 \mathrm{~nm}$, que não corresponde à máxima absorção do licopeno, a sensibilidade do método não foi afetada, fato também observado no trabalho de Müller et al. ${ }^{15}$

Tabela 1. Valores utilizados na avaliação da seletividade, efeito da matriz

\begin{tabular}{lccc}
\hline Licopeno $^{\mathrm{a}}$ & Licopeno $^{\mathrm{b}}$ & $\beta$-caroteno & \\
$\mathrm{n} 1=3$ & $\mathrm{n} 2=3$ & $\mathrm{n} 1=3$ & $\mathrm{n} 2=3$ \\
$\bar{X} 1=13,65$ & $\overline{X 2}=14,46$ & $\bar{X} 1=6,40$ & $\overline{X 2}=6,92$ \\
$\mathrm{~s} 1=0,12$ & $\mathrm{~s} 2=0,08$ & $\mathrm{~s} 1=0,34$ & $\mathrm{~s} 2=0,06$ \\
\hline
\end{tabular}

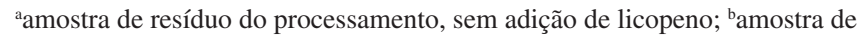
resíduo do processamento com adição de $2,02 \mathrm{mg} 100 \mathrm{~g}^{-1}$ de licopeno; ‘polpa de tomate (produto final), sem adição de $\beta$-caroteno; 'polpa de tomate (produto final), com adição de $0,55 \mathrm{mg} 100 \mathrm{~g}^{-1}$ de $\beta$-caroteno; $\mathrm{n} 1$ e $\mathrm{n} 2$, tamanho das amostras 1 e 2; $X 1$ e $X 2$, médias das respostas dos analitos; s1 e s2, estimativas de desvios-padrão da resposta dos analitos

\section{Sensibilidade}

Os resultados obtidos no teste de sensibilidade podem ser observados na Tabela 2. As curvas analíticas foram avaliadas pelo teste $t$-Student e os valores $t$ calculados foram 17,776 para licopeno e 31,559 para $\beta$-caroteno. O valor tabelado $t$-Student para $n-2$ graus de liberdade com $95 \%$ de probabilidade é $2,776,{ }^{14}$ portanto, a correlação foi significativa para a faixa avaliada. A faixa de linearidade utilizada no estudo ficou próxima à relatada em trabalhos prévios $(0,05$ e 4,0 $\left.\mu \mathrm{g} \mathrm{mL} \mathrm{L}^{-1}\right) \cdot{ }^{16,17} \mathrm{O}$ coeficiente de correlação determinado para licopeno e $\beta$-caroteno foi inferior ao relatado nos mesmos trabalhos $(0,999$ $1,000),{ }^{15-17}$ embora a correlação tenha sido considerada significativa pelo teste $t$-Student.

Tabela 2. Parâmetros de linearidade, limites de detecção (LD) e de quan-

\begin{tabular}{|c|c|c|}
\hline & Licopeno & $\beta$-caroteno \\
\hline $\begin{array}{l}\text { RL: Curva analítica } \\
\left(\mu \mathrm{g} \text { área }^{-1}\right)\end{array}$ & Área $=2.361 .856 c+170.405$ & Área $=3.015 .646 c-97.369$ \\
\hline $\begin{array}{l}\text { RL: Curva LD e LQ } \\
\left(\mu \mathrm{g} \text { área }^{-1}\right)\end{array}$ & Área $=616.404 \mathrm{c}-39.953$ & Área $=3000000 c-72042$ \\
\hline $\begin{array}{l}\text { RL: Curva LD e LQ } \\
\left(\mu \mathrm{g} \mathrm{s}^{-1}\right)\end{array}$ & Área $=98.489 \mathrm{c}-7.477$ & Área $=126.022 \mathrm{c}-1.1946$ \\
\hline $\begin{array}{l}\text { Coeficiente de cor- } \\
\text { relação (r) }\end{array}$ & 0,9937 & 0,9980 \\
\hline $\mathrm{LD}\left(\mu \mathrm{g} \mathrm{mL} L^{-1}\right)$ & 0,04 & 0,02 \\
\hline $\mathrm{LD}\left(\mathrm{mg} 100 \mathrm{~g}^{1}\right)$ & 4,19 & 0,23 \\
\hline $\mathrm{LQ}\left(\mu \mathrm{g} \mathrm{mL} \mathrm{L}^{-1}\right)$ & 0,08 & 0,03 \\
\hline $\mathrm{LQ}\left(\mathrm{mg} 100 \mathrm{~g}^{1}\right)$ & 7,70 & 0,26 \\
\hline
\end{tabular}
tificação (LQ), e valores de LD e LQ determinados pelo método proposto

RL, equação de regressão linear; c, concentração; s, estimativa de desvio padrão das áreas obtidas nas três injeções de cada concentração.

O limite de detecção para licopeno, determinado pelo método proposto, foi $0,04 \mu \mathrm{g} \mathrm{mL}{ }^{-1}$, semelhante ao encontrado por Hart e Scott $\left(0,035 \mu \mathrm{g} \mathrm{mL}^{-1}\right)^{16}$ e superior ao relatado por Müller et al. ${ }^{15}(0,01 \mu \mathrm{g}$ $\left.\mathrm{mL}^{-1}\right)$ e Dias et al. ${ }^{17}\left(0,02 \mu \mathrm{g} \mathrm{mL} \mathrm{L}^{-1}\right)$. O limite de quantificação encontrado foi $0,08 \mu \mathrm{g} \mathrm{mL}^{-1}$, superior ao relatado pelos mesmos autores $\left(0,05 \mu \mathrm{g} \mathrm{mL} \mathrm{m}^{-1}\right){ }^{15,17}$

O limite de detecção encontrado para $\beta$-caroteno foi $0,02 \mu \mathrm{g} \mathrm{mL}{ }^{-1}$, semelhante ao relatado em trabalhos prévios, ${ }^{17,18}$ e cerca de $50 \%$ inferior ao valor encontrado por Hart e Scott $(0,045 \mu \mathrm{g} \mathrm{mL}-1) .{ }^{16} \mathrm{O}$ limite de quantificação determinado pelo método proposto $\left(0,03 \mu \mathrm{g} \mathrm{mL}^{-1}\right)$ ficou cerca de $50 \%$ inferior ao relatado por Dias et al. ${ }^{17}\left(0,055 \mu \mathrm{g} \mathrm{mL}^{-1}\right)$. 


\section{Precisão}

Os coeficientes de variação encontrados, para as cinco repetições analíticas, e as áreas das cinco injeções do padrão analítico, foram 4 e $5 \%$ para o licopeno e $\beta$-caroteno, respectivamente (Tabela 3 ). Os coeficientes de variação encontrados foram menores que os valores obtidos pela equação de Horwitz ${ }^{12}$ (Equação 6), portanto o método e o sistema analítico apresentaram precisão adequada. Valores semelhantes foram encontrados em trabalhos anteriores (4 e 6\%). ${ }^{15}$

Tabela 3. Valores encontrados na precisão do método e do sistema cromatográfico para licopeno e $\beta$-caroteno

\begin{tabular}{|c|c|c|c|c|}
\hline \multirow[b]{2}{*}{ Dados } & \multicolumn{2}{|c|}{ Licopeno } & \multicolumn{2}{|c|}{$\beta$-caroteno } \\
\hline & 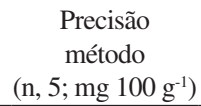 & $\begin{array}{c}\text { Precisão } \\
\text { sistema } \\
(\mathrm{n}, 5 ; \text { área })\end{array}$ & 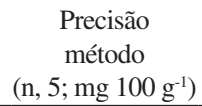 & $\begin{array}{c}\text { Precisão } \\
\text { sistema } \\
\text { (n, 5; área) }\end{array}$ \\
\hline Valor médio & 104 & 6699721 & 6,40 & 2727400 \\
\hline $\mathrm{s}$ & 4 & 256477 & 0,34 & 46485 \\
\hline $\mathrm{CV}(\%)$ & 4 & 4 & 5 & 2 \\
\hline CV Max (\%) & \multicolumn{2}{|l|}{6} & \multicolumn{2}{|l|}{13} \\
\hline
\end{tabular}

n, número de replicatas; $\mathrm{s}$, estimativa de desvio padrão; $\mathrm{CV}$, coeficiente de variação; CV Max (\%), coeficiente de variação obtido pela equação de Horwitz.

\section{Exatidão}

Os teores médios de carotenoides obtidos nos testes de recuperação foram 1,82 e 2,62 mg $100 \mathrm{~g}^{-1}$ para licopeno e 0,52 e 0,85 mg $100 \mathrm{~g}^{-1}$ para $\beta$-caroteno. Descontando a concentração dos pigmentos naturalmente presente nas amostras, as recuperações médias foram de 90,65 e 93,76\%, respectivamente (Tabela 4). Os valores calculados de $t$ foram 2,060 e 1,382 para licopeno e $\beta$-caroteno, respectivamente, e esses valores foram comparados com o valor tabelado ( $t$-Student) com $n-1$ graus de liberdade para uma probabilidade de $95 \%$ ( $t$ tabelado $_{(95,5)}$ igual a 2,571). ${ }^{14}$ Os resultados indicaram que o método apresenta exatidão adequada com $95 \%$ de confiança.

Tabela 4. Valores utilizados na avaliação da exatidão para licopeno e $\beta$-caroteno

\begin{tabular}{|c|c|c|c|c|}
\hline \multirow{2}{*}{ Dados } & \multicolumn{2}{|c|}{ Licopeno } & \multicolumn{2}{|c|}{$\beta$-caroteno } \\
\hline & A & B & A & B \\
\hline Adicionado (mg $100 \mathrm{~g}^{-1}$ ) & 2,02 & 2,89 & 0,55 & 0,91 \\
\hline Recuperação média (mg $100 \mathrm{~g}^{-1}$ ) & 1,82 & 2,62 & 0,52 & 0,85 \\
\hline $\mathrm{s}$ & 0,08 & 0,15 & 0,06 & 0,07 \\
\hline $\mathrm{CV}(\%)$ & 5 & 6 & 12 & 8 \\
\hline CV $\operatorname{Max}(\%)$ & 15 & 16 & 17 & 17 \\
\hline Recuperação (\%) & 90,4 & 90,9 & 94,7 & 92,9 \\
\hline Recuperação média (\%) & \multicolumn{2}{|c|}{90,7} & \multicolumn{2}{|c|}{93,8} \\
\hline S & \multicolumn{2}{|c|}{0,38} & \multicolumn{2}{|c|}{1,27} \\
\hline $\mathrm{CV}(\%)$ & \multicolumn{2}{|c|}{0,42} & \multicolumn{2}{|c|}{1,35} \\
\hline CV Max (\%) & \multicolumn{2}{|c|}{2,03} & \multicolumn{2}{|c|}{2,02} \\
\hline
\end{tabular}

$\mathrm{s}=$ estimativa de desvio padrão; $\mathrm{CV}=$ coeficiente de variação; CV Max $(\%)$ = coeficiente de variação obtido pela equação de Horwitz.

Valores médios de recuperação entre 94 e 109\% para licopeno e 84 e $101 \%$ para $\beta$-caroteno em tomates foram relatados em trabalhos prévios $^{19,20}$ e são próximos aos valores encontrados neste trabalho.

\section{Estimativa de incerteza}

Para identificar as fontes de incertezas utilizou-se o diagrama de causa e efeito (Figura 2), onde estão relacionadas as principais fontes de incertezas associadas a cada etapa analítica. A concentração dos carotenoides foi afetada pelas seguintes fontes: diluição da amostra $\left(\right.$ Dil $\left._{\mathrm{am}}\right)$, área da amostra $\left(\mathrm{A}_{\mathrm{am}}\right)$, concentração do padrão $\left(\mathrm{C}_{\mathrm{p}}\right)$, área do padrão $\left(\mathrm{A}_{\mathrm{p}}\right)$ e massa da amostra $\left(\mathrm{m}_{\mathrm{am}}\right)$, conforme Equação 9.

\section{Padrão}

injeção

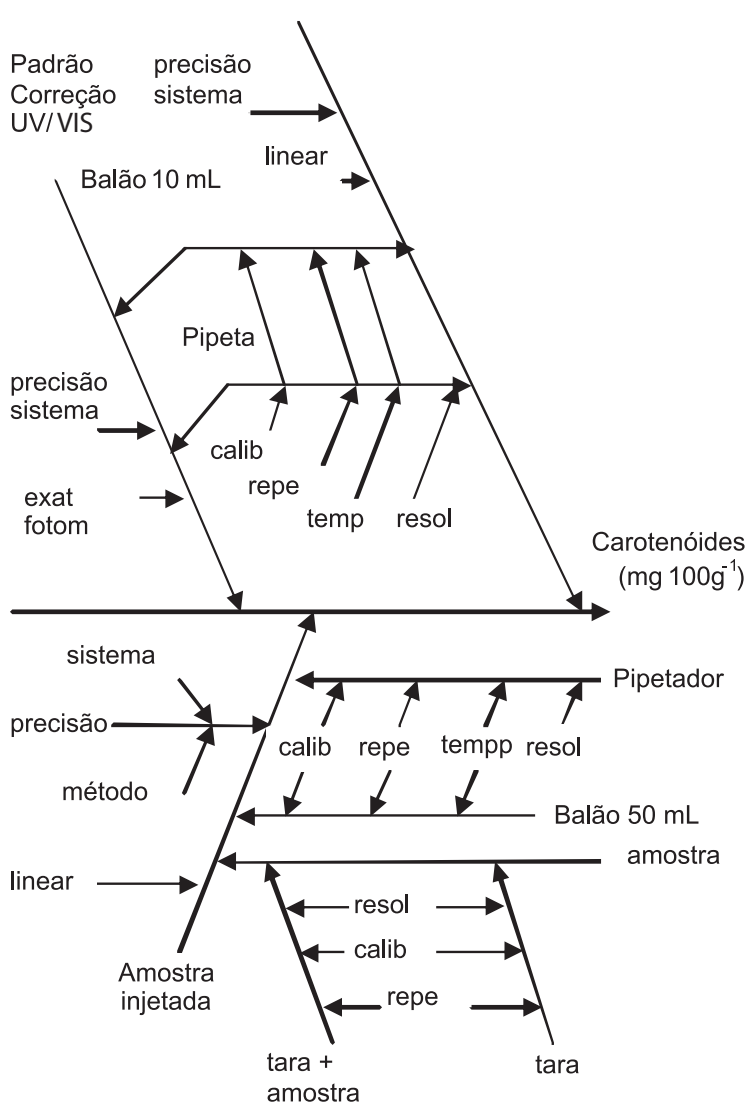

Figura 2. Diagrama de causa e efeito com os dados utilizados no cálculo de incerteza na determinação por CLAE de licopeno e $\beta$-caroteno em polpa de tomate liofilizada. linear $=$ linearidade, calib $=$ calibração $;$ repe $=r e-$ petitividade, temp = temperatura; resol = resolução, exat fotom $=$ exatidão fotométrica

O cálculo da incerteza da linearidade foi baseado no método dos mínimos quadrados ${ }_{15}^{15}$ considerado a equação da reta como $\mathrm{A}_{\mathrm{j}}=\mathrm{c}_{\mathrm{i}} \mathrm{B}_{1}$ $+\mathrm{B}_{0} \mathrm{Na}$ determinação da incerteza expandida foi utilizado o fator de abrangência $\mathrm{K}$ igual a 2 .

A incerteza combinada para licopeno, para uma concentração

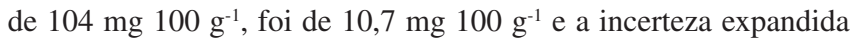
foi de $21 \mathrm{mg} 100 \mathrm{~g}^{-1}(\mathrm{~K}=2)$, que corresponde a uma incerteza expandida de $21 \%$ da concentração estudada. $O \beta$-caroteno apresentou incerteza combinada, para uma concentração de $6,4 \mathrm{mg} 100 \mathrm{~g}^{-1}$, de $0,75 \mathrm{mg} 100 \mathrm{~g}^{-1}$ e incerteza expandida de $1,5 \mathrm{mg} 100 \mathrm{~g}^{-1}(\mathrm{~K}=2)$, que corresponde a uma incerteza de $23 \%$ da concentração estudada. A contribuição, em percentagem, de cada uma das fontes de incertezas está apresentada na Figura 3.

Dias et al. ${ }^{17}$ relataram incerteza expandida próxima à encontrada neste trabalho para licopeno $(25 \%),{ }^{17}$ e superior para $\beta$-caroteno (14\%). A maior contribuição na incerteza relativa, para os dois analitos, foi dada pelos pipetadores utilizados nas diluições dos padrões e amostras (Figura 3), como observado em trabalho prévio. ${ }^{17} \mathrm{Na}$ determinação de licopeno os pipetadores contribuíram com $60 \%$ da incerteza total e na determinação do $\beta$-caroteno com $42 \%$. 


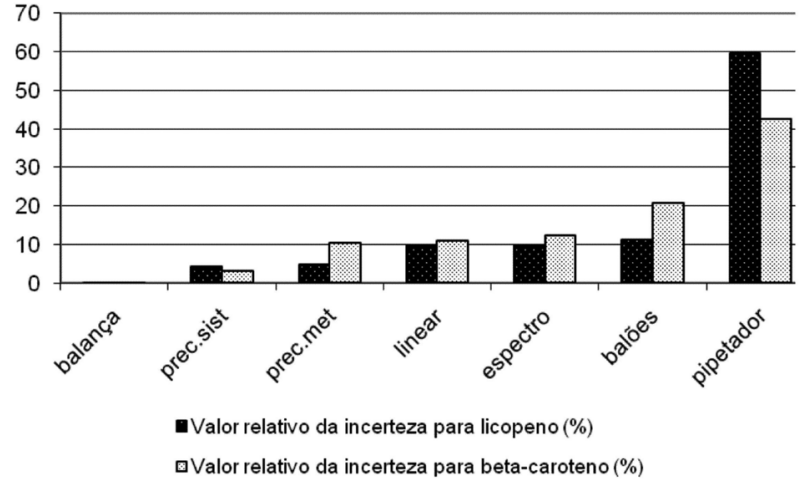

Figura 3. Contribuição percentual de cada incerteza para licopeno e $\beta$-caroteno

\section{CONCLUSÃO}

O método validado apresentou linearidade na faixa de concen-

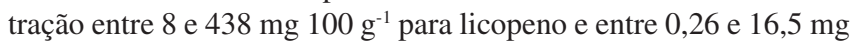
$100 \mathrm{~g}^{-1}$ para $\beta$-caroteno, com boa precisão e exatidão. A incerteza expandida $(K=2)$ foi de aproximadamente $22 \%$ para uma concen-

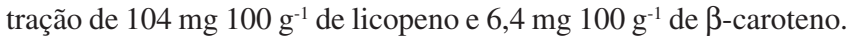

\section{AGRADECIMENTOS}

À FAPESP pelo auxílio financeiro e à Predilecta Alimentos Ltda pelas amostras utilizadas no estudo.

\section{REFERÊNCIAS}

1. http://www.cepea.esalq.usp.br/hfbrasil/edicoes/58/mat_capa.pdf, acessada em Março 2009 e Julho 2010.

2. http://www.cpafro.embrapa.br/embrapa/Artigos/tomateiro.htm, acessada em Março 2009 e Julho 2010.

3. Pedro, A. M. K.; Dissertação de Mestrado, Universidade Estadual de Campinas, Brasil, 2004.
4. Kavanaugh, C. J.; Trumbo, P. R.; Ellwood, K. C. J.; Natl. Cancer Inst. 2007, 99, 1074

5. http://www.nal.usda.gov/fnic/DRI//DRI_Vitamin_C/R1-16_150.pdf, acessada em Março 2009 e Julho 2010.

6. http://allchemy.iq.usp.br/pub/metabolizando/Beta021115.pdf, acessada em Julho 2010.

7. Davies, B. H. In Chemistry and Biochemistry of Plant Pigments; Goodwing, T. W., ed.; Academic Press: New York, 1976, chap.19.

8. Carvalho, P. R. N.; Collins, C. A.; Rodriguez-Amaya, D. B.; Chromatographia 1992, 33, 133.

9. Instituto Nacional de metrologia, Normalização e Qualidade Industrial; Orientação sobre validação de métodos de ensaios químicos, $D O Q$ CGCRE-008, revisão 02 de 06/2007.

10. http://www.fao.org/docrep/w5975e/w5975e09.htm, acessada em Abril 2009 e Julho 2010; http://www.fao.org/docrep/meeting/005/w4873e/ w4873e0m.htm, acessada em Abril 2009 e Julho 2010.

11. Quattrocchi, O. A.; Andrizzi, S. A.; Laba, R. F.; Introdutión a la HPLC Aplicación y Práctic, Artes Gráficas Farro SA: Buenos Aires, 1992.

12. Horwitz, W.; Anal. Chem. 1982, 54, 67A.

13. Instituto Nacional de metrologia, Normalização e Qualidade Industrial; Vocabulário internacional de termos fundamentais e gerais de metrologia, $3^{\text {a }}$ ed., Rio de Janeiro, 2003.

14. Massart, D. L.; Vandeginste, B. G. M.; Deming, S. N.; Micotte, Y.; Kaufman, L.; Chemometrics: a textbook, Elsevier Science Publishers Company Inc.: New York, 1988.

15. Müller, A.; Pietsch, B.; Faccin, N.; Schierle, J.; J. AOAC Int. 2008, 6 , 1284

16. Hart, D. J.; Scott, K. J.; Food Chem. 1995, 54, 101.

17. Dias, M. G.; Camões, M. F. G. F. C.; Oliveira, L.; Food Chem. 2008 , 109, 815 .

18. Sérino, S.; Gomez, L.; Costagliola, G.; Gautier, H.; J. Agric. Food Chem. 2009, 57, 8753 .

19. Barba, A. I. O.; Hurtado, M. C.; Mata, M. C. S.; Ruiz, V. F.; de Tejada, M. L. S.; Food Chem. 2006, 95, 328.

20. 19. Lin, C. H.; Chen, B. H.; J. Chromatogr., A 2003, 1012, 103. 